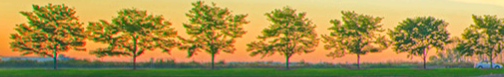

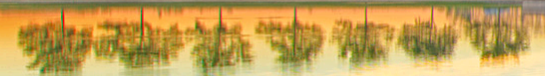

\section{Variational quantum simulation of interacting bosons on NISQ devices}

Andy C. Y. Li

Kavli ACP Spring Workshop: Intersections QIS/HEP 20 May 2019

This manuscript has been authored by Fermi Research Alliance, LLC under Contract No. DE-AC02-07CH11359 with the U.S. Department of Energy, Office of Science, Office of High Energy Physics

This document has been authored by Fermi Research Alliance, LLC under Contract No. DE-AC02-07CH11359 with the U.S. Department of Energy, Office of Science, Office of High Energy Physics.

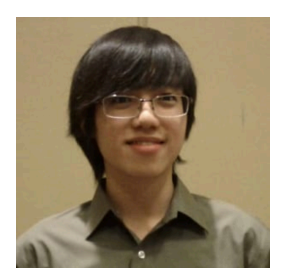

Andy C. Y. Li

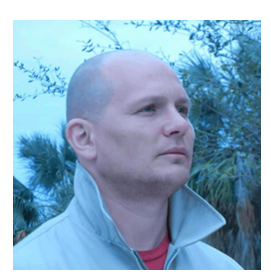

Alex Macridin

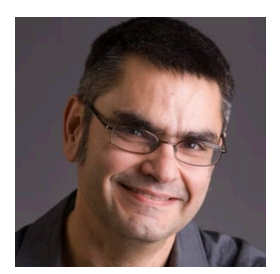

Panagiotis Spentzouris 


\section{Outline}

- Noisy Intermediate-Scale Quantum (NISQ) devices

- Quantum-classical hybrid variational algorithms

- Variational quantum eigensolver (VQE) of interacting bosons

- Proof-of-principle experiment of a 3-qubit implementation

- Open questions about scalability 


\section{Digital quantum simulation}

- "Nature isn't classical, dammit, and if you want to make a simulation of nature, you'd better make it quantum mechanical" Richard Feynman (1982)

- Digital: all operations are represented by qubit gates

- Time evolution, quantum phase estimation, quantum annealing, ...

- Targets: highly entangled quantum states, non-perturbative system dynamics, ...

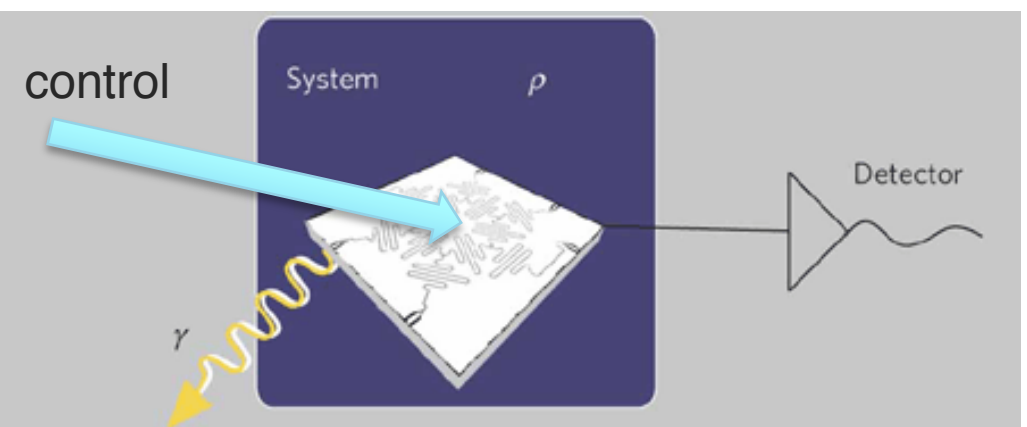

Photon leakage,

qubit relaxation

Environment

A

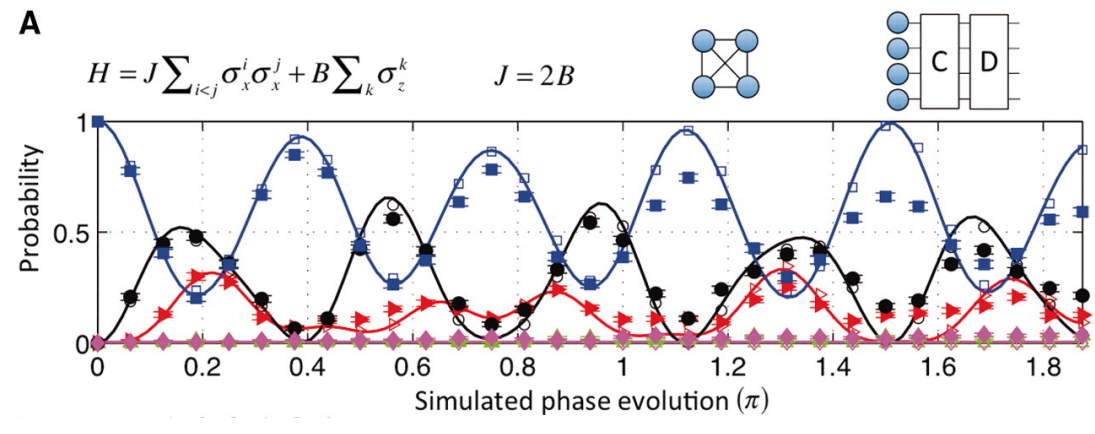

Science 334.6052 (2011): 57-61 


\section{Challenges: noise and control error}

- Decoherence: relaxation, pure dephasing, correlated noise, ...

$\rightarrow$ device loses 'quantumness' after a limited coherence time

- Control error: inaccurate gate implementation due to imperfect calibration, qubit drift, ...

$\rightarrow$ reliable result only within a limited number of gate operations

- Only shallow circuits can be reliably implemented in the near future
Relaxation $\left(T_{1}\right)$
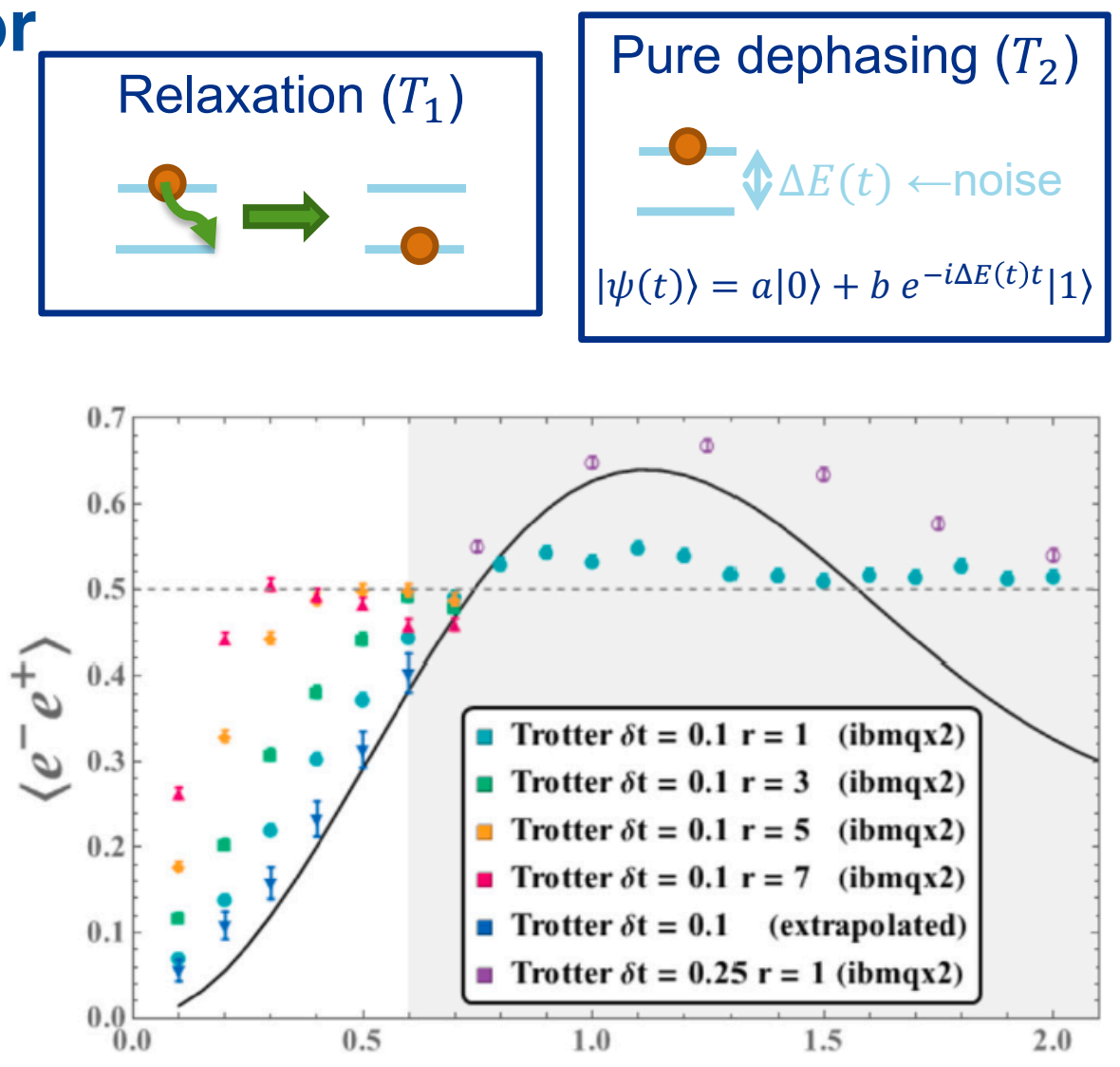

Martin Savage's group: scaled time PRA 98, 032331 (2018) 


\section{Superconducting qubit coherence}
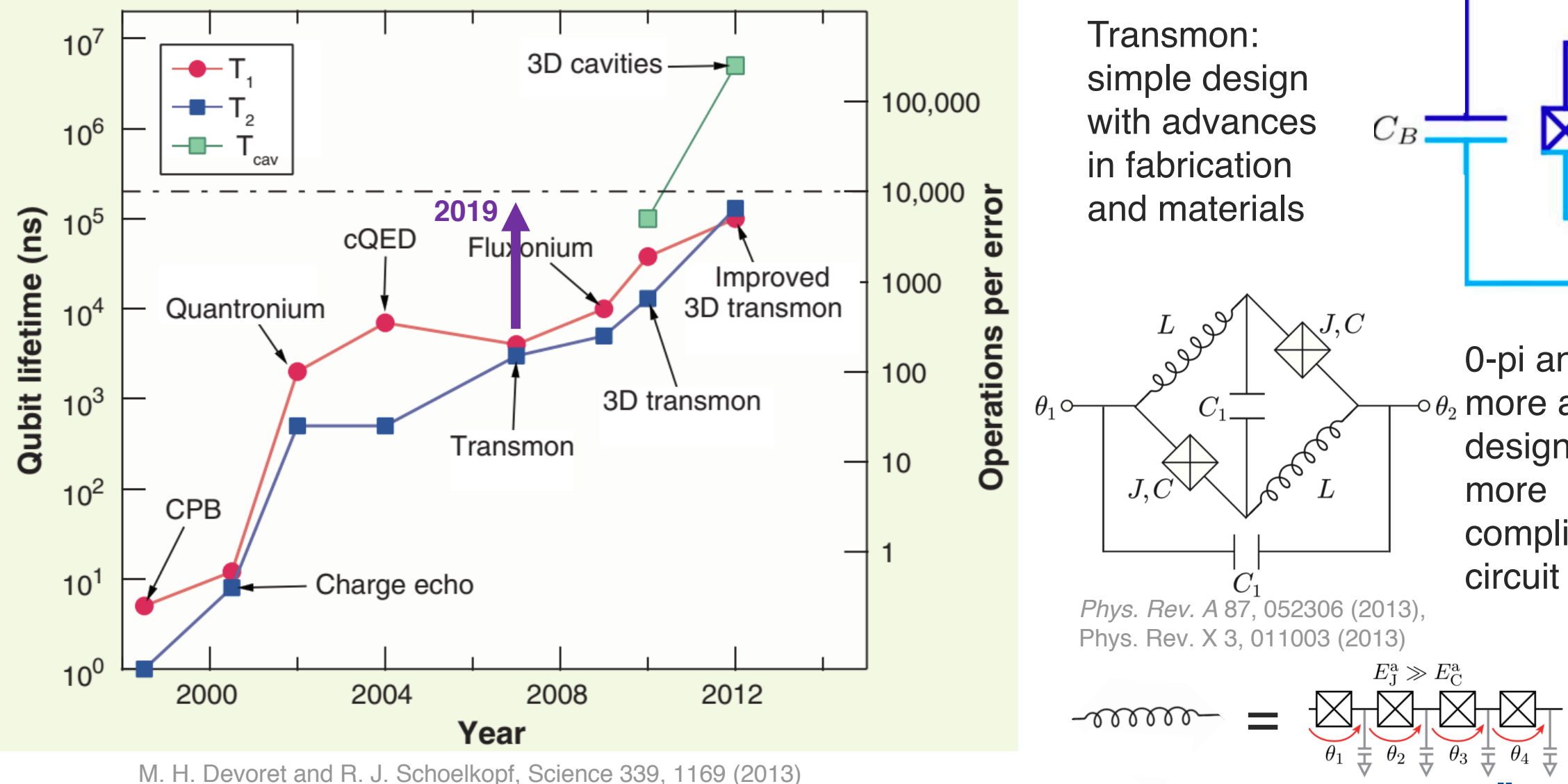

$C_{J}, E_{J}$ $\Phi$

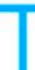




\section{Quantum computing for NISQ devices}

https://qutech.nl/ majorana-trilogy completed/

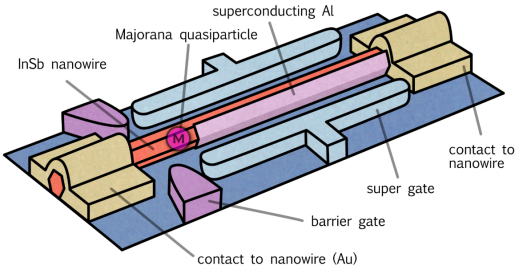

Fault-tolerant qubit

- unclear path to realize

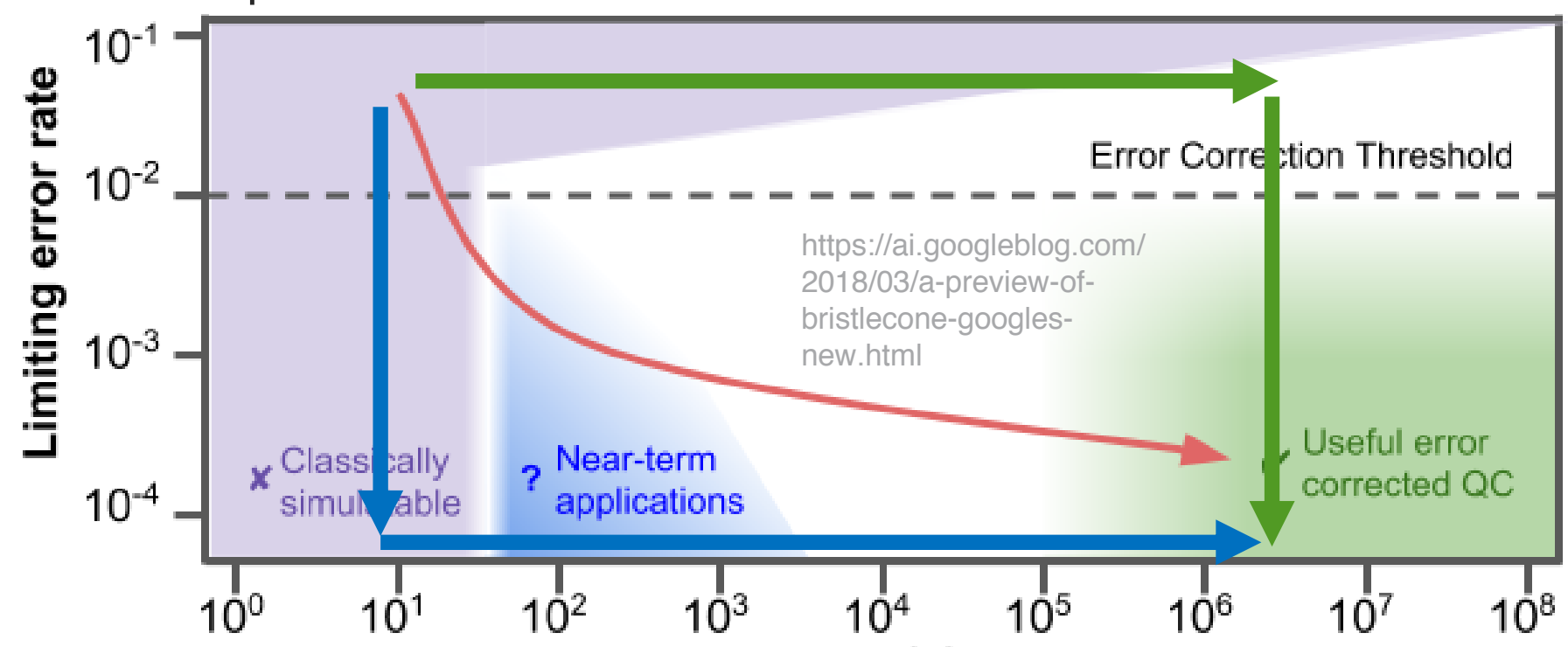

Number of Qubits
D-wave quantum annealing

- unclear quantum advantage

Analog open-system simulation

- very limited applications

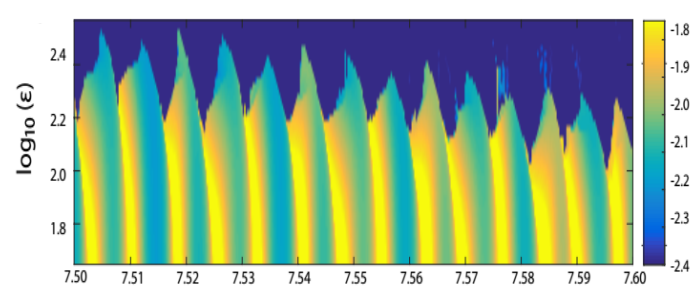

Phys. Rev. X 7 011016 (2017)

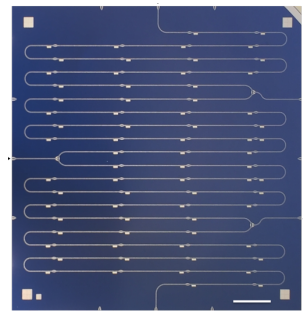

Noisy IntermediateScale Quantum (NISQ) devices: 100 pre-threshold qubits capable for shallow circuits 


\section{Quantum-classical hybrid variational algorithms}

- Quantum Approximate Optimization Algorithm (QAOA)

- Approximated solutions for combinatorial optimization problems through a series of classically optimized gate operations

- Quantum kernel method

- Support vector machine (SVM) with kernel function evaluated by quantum devices

- Quantum autoencoder

- encoding in Hilbert space with encoder trained classically

- Variational quantum eigensolver (VQE)

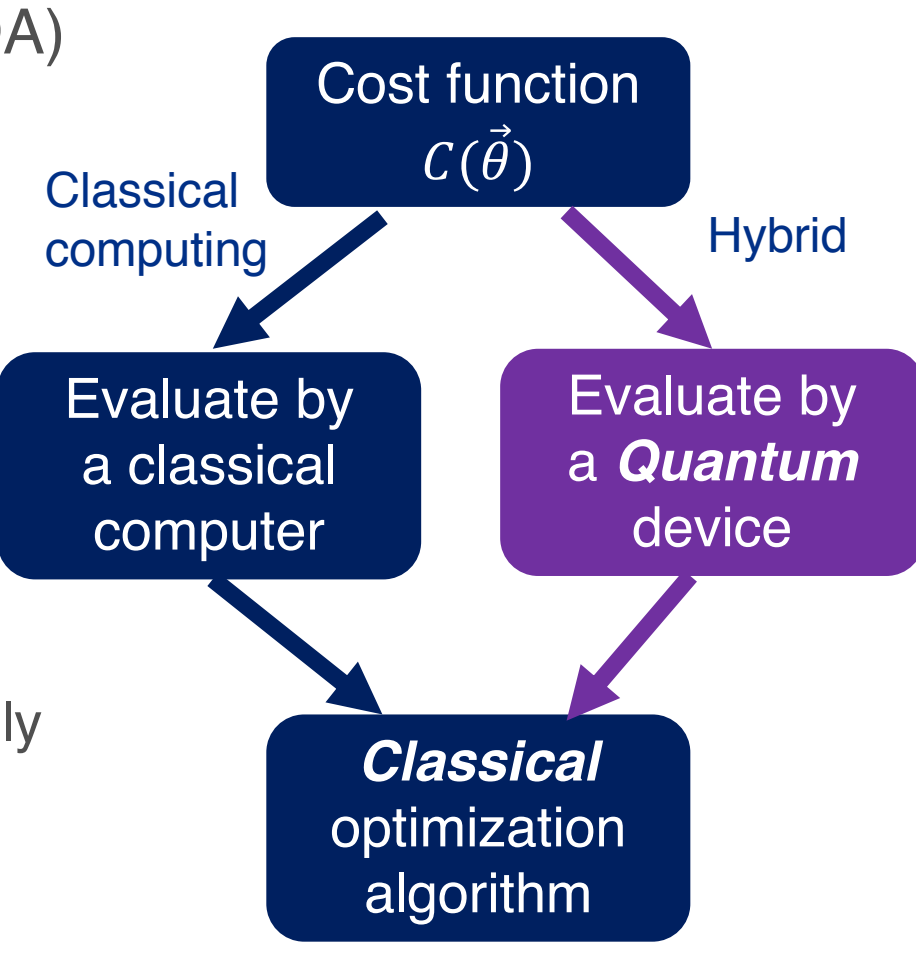

- Variational ansatz represented by a list of quantum gate and optimized by a classical optimizer 


\section{Why hybrid variational algorithms?}

- Relatively shallow circuit

$\mathrm{R}_{\mathrm{Y}}\left(\theta_{0}\right)-\mathrm{R}_{\mathrm{Z}}\left(\theta_{1}\right) \cdot \mathrm{R}_{\mathrm{Y}}\left(\theta_{6}\right)-\mathrm{R}_{\mathrm{Z}}\left(\theta_{7}\right)$

$-\mathrm{R}_{\mathrm{Y}}\left(\theta_{2}\right)-\mathrm{R}_{\mathrm{Z}}\left(\theta_{3}\right) \cdot \mathrm{R}_{\mathrm{Y}}\left(\theta_{8}\right)-\mathrm{R}_{\mathrm{Z}}\left(\theta_{9}\right) \cdot \mathrm{R}_{\mathrm{Y}}\left(\theta_{10}\right)-\mathrm{R}_{\mathrm{Z}}\left(\theta_{11}\right)$
$-\mathrm{R}_{\mathrm{Y}}\left(\theta_{4}\right)-\mathrm{R}_{\mathrm{Z}}\left(\theta_{5}\right)$
$-\mathrm{R}_{\mathrm{Y}}\left(\theta_{12}\right)-\mathrm{R}_{\mathrm{Z}}\left(\theta_{13}\right)$

- Tolerant to control errors (coherent rotation angle errors )

- Quantum advantage?

- Heuristic and most likely problem-specific

- Possible sources of quantum advantage

- Quantum tunneling (QAOA)

- Hilbert space size: $2^{N}$ (Quantum machine learning)

- Natural way to evaluate $\langle H\rangle, \ldots$ (VQE)

Information and Software Technology

Volume 41, Issue 2, 25 January 1999, Pages 107-117

Heuristic principles for the design of artificial neural networks

Steven Walczak ${ }^{\circ} \cap^{\otimes}$, Narciso Cerpa $^{b}$

\begin{tabular}{|c|c|c|c|c|c|c|c|c|c|}
\hline \multicolumn{10}{|c|}{ Computer Science $>$ Computation and Language } \\
\hline \multirow{2}{*}{\multicolumn{10}{|c|}{ BERT: Pre-training of Deep Bidirectional Transformers for Language Understandin }} \\
\hline & & & & & & & & & \\
\hline \multirow[t]{2}{*}{ System } & MNLI-(m/mm) & QQP & QNLI: & SST-2 & CoLA & STS-B & MRPC & RTE & Average \\
\hline & $392 \mathrm{k}$ & $363 \mathrm{k}$ & $108 \mathrm{k}$ & $67 \mathrm{k}$ & $8.5 \mathrm{k}$ & $5.7 \mathrm{k}$ & $3.5 \mathrm{k}$ & $2.5 \mathrm{k}$ & - \\
\hline Pre-OpenAI SOTA & $80.6 / 80.1$ & 66.1 & 82.3 & 93.2 & 35.0 & 81.0 & 86.0 & 61.7 & 74.0 \\
\hline BiLSTM+ELMo+Attn & $76.4 / 76.1$ & 64.8 & 79.9 & 90.4 & 36.0 & 73.3 & 84.9 & 56.8 & 71.0 \\
\hline OpenAI GPT & $82.1 / 81.4$ & 70.3 & 88.1 & 91.3 & 45.4 & 80.0 & 82.3 & 56.0 & 75.2 \\
\hline BERT $_{\text {BASE }}$ & $84.6 / 83.4$ & 71.2 & 90.1 & 93.5 & 52.1 & 85.8 & 88.9 & 66.4 & 79.6 \\
\hline BERT $_{\text {LARGE }}$ & $86.7 / 85.9$ & 72.1 & 91.1 & 94.9 & 60.5 & 86.5 & 89.3 & 70.1 & 81.9 \\
\hline
\end{tabular}




\section{Variational quantum eigensolver (VQE)}

Encoding: system representation qubits
Variational ansatz: parameterized circuit to prepare the trial state

Update

Classical optimization algorithm
- Ground-state properties

- Long-time scale responses
Noisy intermediate Scale Quantum (NISQ) devices

Efficient

measurement

Trial state's energy 


\section{VQE applications in quantum chemistry}

\section{LETTER}

doi:10.1038/nature23879

Hardware-efficient variational quantum eigensolver for small molecules and quantum magnets

Abhinav Kandala ${ }^{1 *}$, Antonio Mezzacapo ${ }^{1 *}$, Kristan Temme ${ }^{1}$, Maika Takita ${ }^{1}$, Markus Brink $^{1}$, Jerry M. Chow ${ }^{1} \&$ Jay M. Gambetta ${ }^{1}$
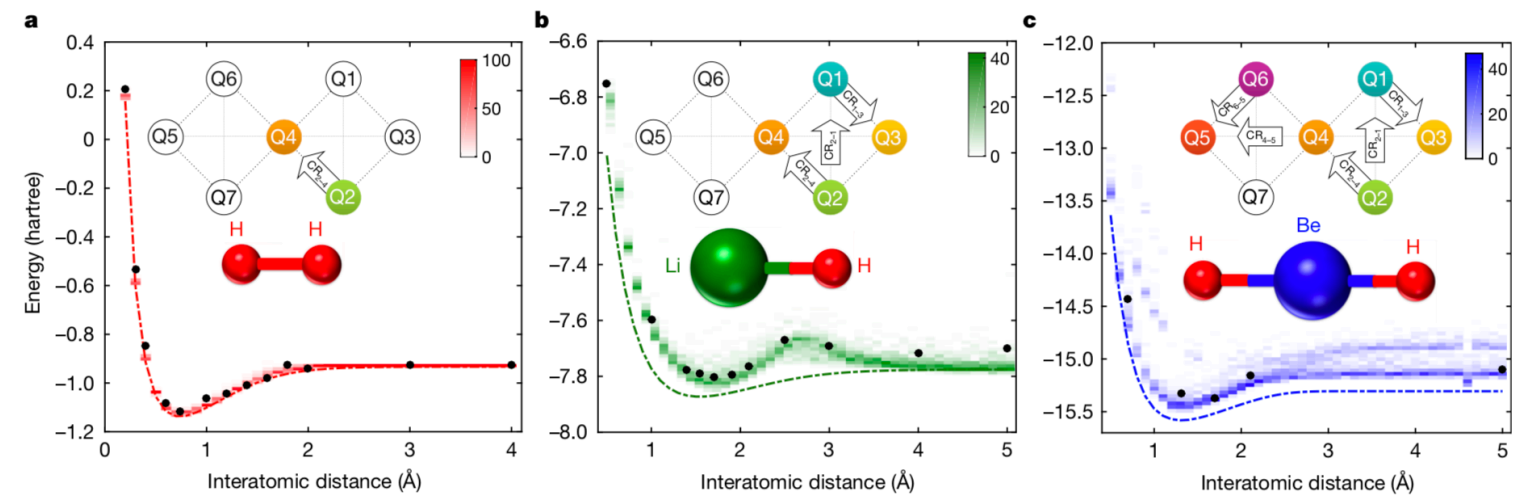

\section{ARTICLE}

Received 9 Dec 2013 | Accepted 27 May 2014 | Published 23 Jul 2014

DOl: 10.1038/ncomms5213 OPEN A variational eigenvalue solver on a photonic quantum processor

Alberto Peruzzo ${ }^{1, *, *}$, Jarrod McClean ${ }^{2, \star}$, Peter Shadbolt ${ }^{1}$, Man-Hong Yung 2,3, Xiao-Qi Zhou', Peter J. Love ${ }^{4}$, Alán Aspuru-Guzik² \& Jeremy L. O'Brien ${ }^{1}$

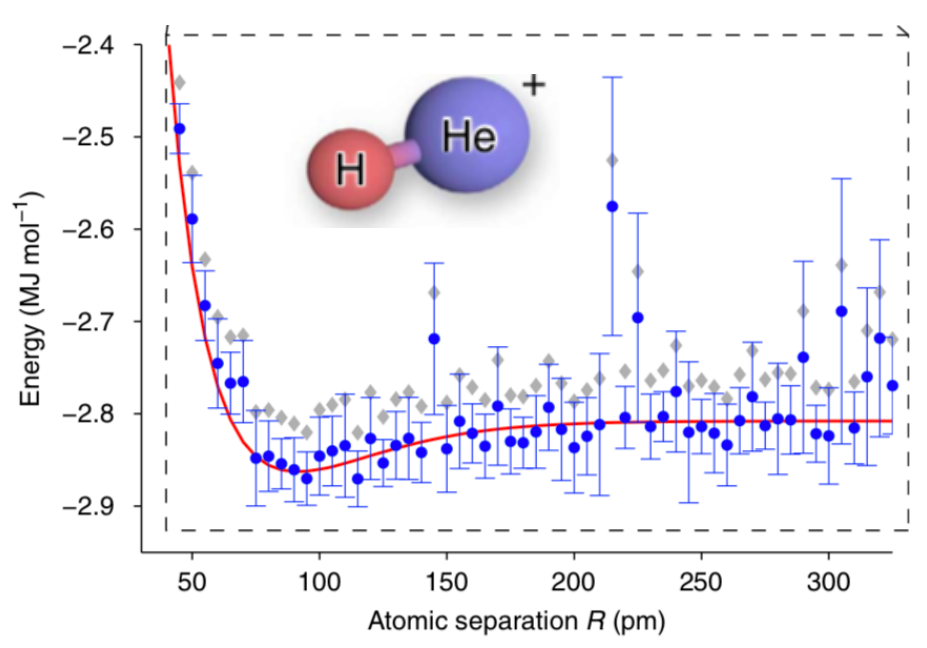




\section{VQE: much less developed for non-fermionic systems}

- Fermions $\leftrightarrow$ Qubits

- Jordan-Wigner transformation
By en:User_talk:S_kliminUpgrade

(New vectorial edition) : Olivier

d'ALLIVY KELLY -

en:File:Polaron_scheme1.jpg, CC BY-SA 4.0,

https://commons. wikimedia.org/w/i ndex. php?curid=8461871
(-)

(2)

(-)

(-)

(2)

- Many models in high-energy and condensedmatter involve non-fermionic degrees of freedom

- Goal: many-body systems with bosons

- light-matter interaction

- electron-phonon coupling

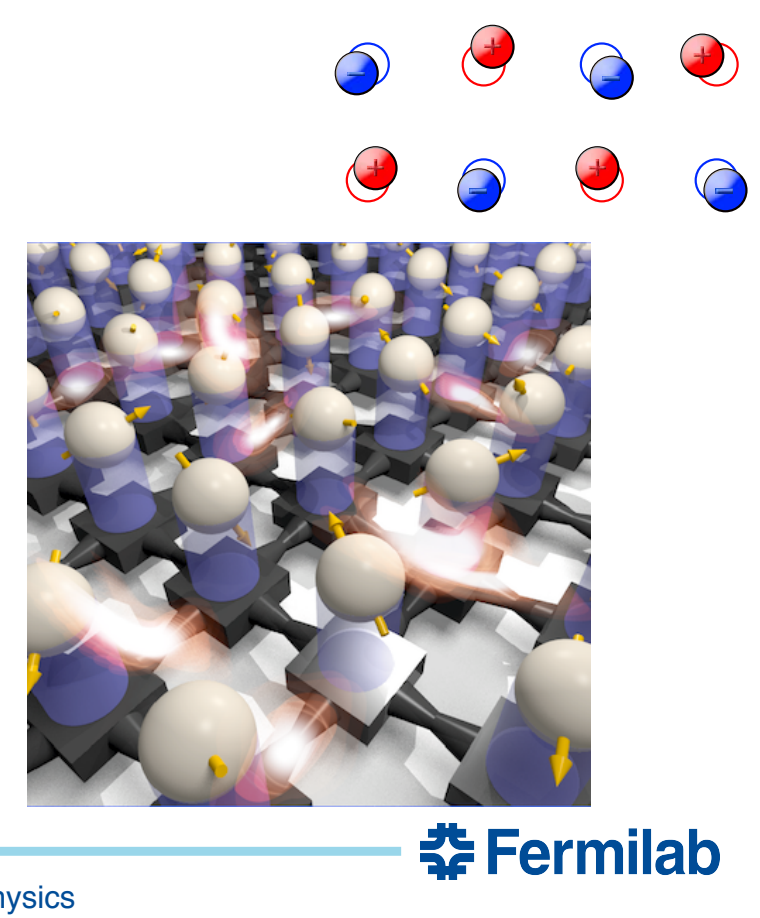




\section{Boson encoding by qubits}

Goal: encode a truncated boson Hilbert space in qubits

\section{Position basis binary encoding}

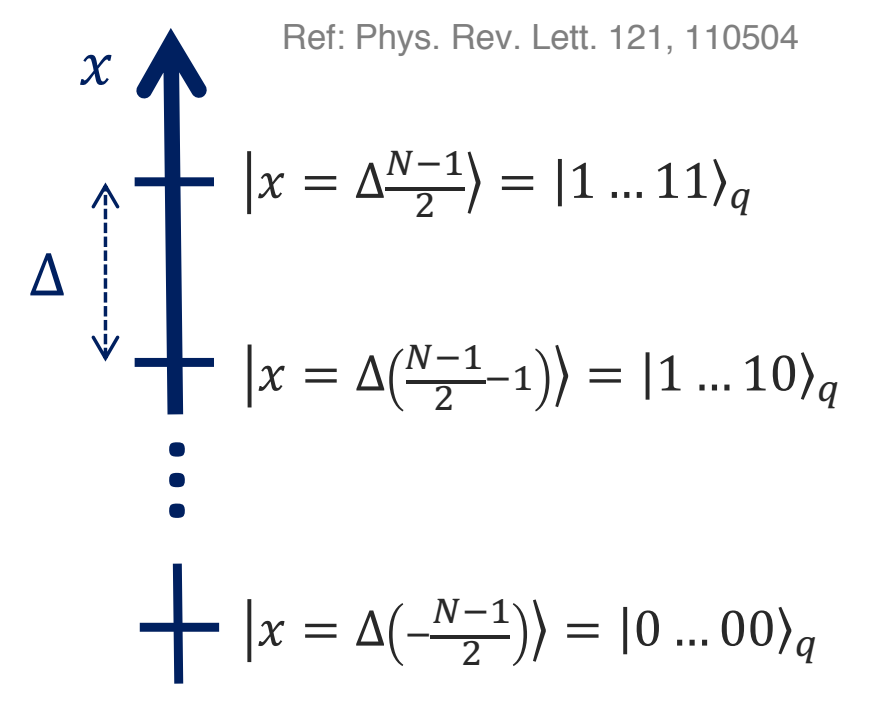

Number basis binary encoding

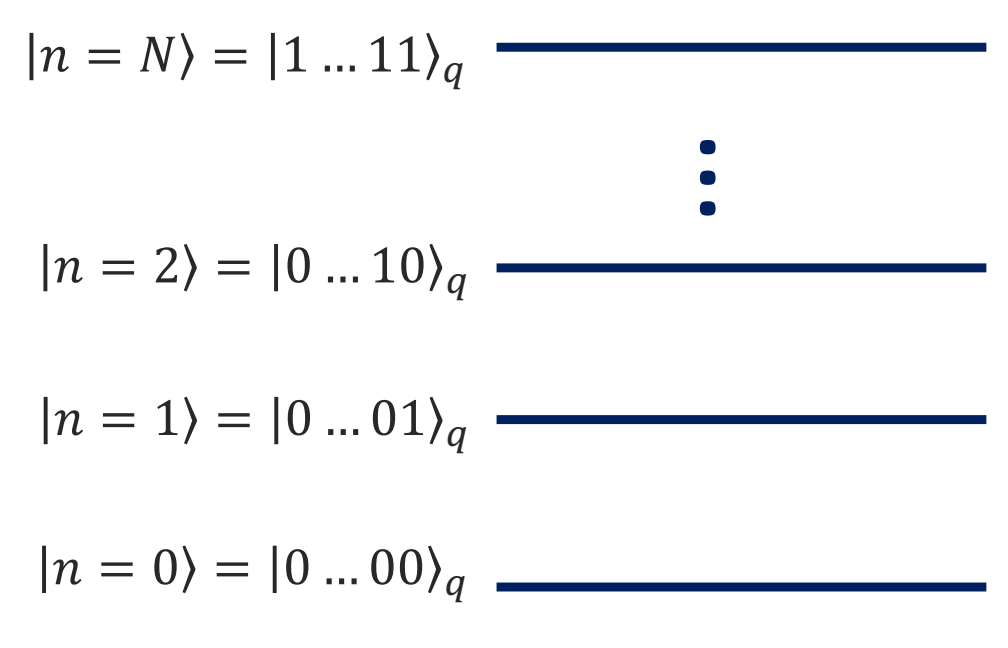




\section{Variational ansatz}

Parameterized gates natively supported by the hardware

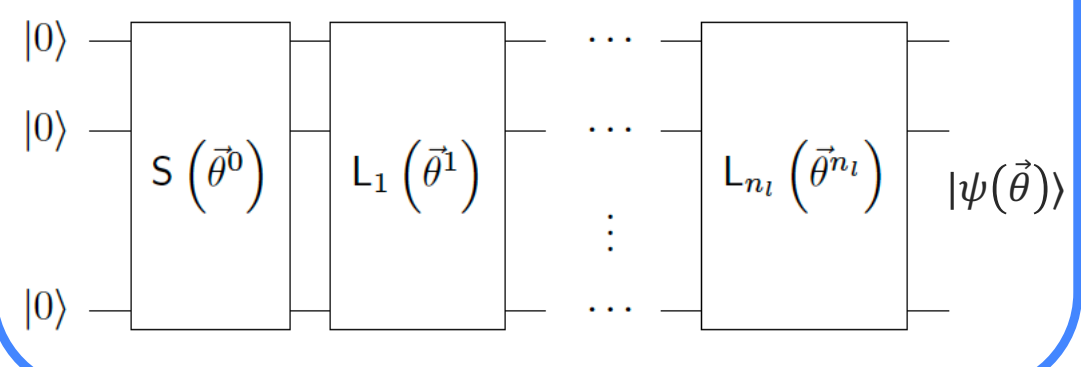

Motivated by adiabatic state transfer

$$
U(\alpha, \theta)=e^{-i \theta\left((1-\alpha) H_{A}+\alpha H_{B}\right)}
$$

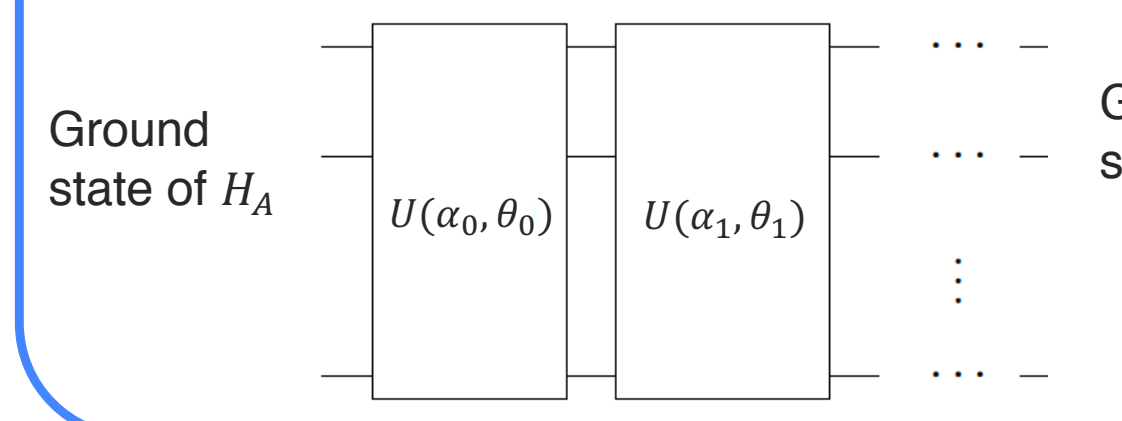

Ground state of $H_{B}$ $|\psi(\vec{\theta})\rangle$
Hardware efficient

Increasing circuit depth
Model motivated

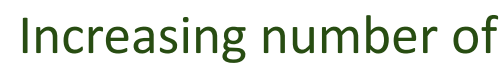

optimization parameters 


\section{Cost function for ground state \& excited states}

Ground-state cost function = trial state's energy

$$
C_{0}(\vec{\theta})=\langle\psi(\vec{\theta})|H| \psi(\vec{\theta})\rangle
$$

Ground state: $\left|\psi_{0}\right\rangle=\operatorname{argmin} C_{0}$

$$
|\psi(\vec{\theta})\rangle
$$

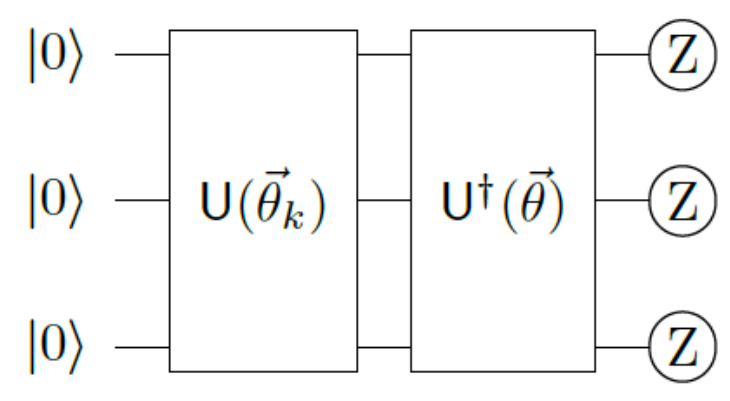

1st-excited state cost function: $C_{1}=\langle\psi(\vec{\theta})|H| \psi(\vec{\theta})\rangle+\underline{\left.\epsilon\left|\left\langle\psi_{0}\right| \psi(\vec{\theta})\right)\right|^{2}}$

1st-excited state: $\left|\psi_{1}\right\rangle=\operatorname{argmin} C_{1}$

Overlap with the ground state $|\psi(\vec{\theta})\rangle$

2nd-excited state cost function: $C_{2}=\langle\psi(\vec{\theta})|H| \psi(\vec{\theta})\rangle+\epsilon\left|\left\langle\psi_{0} \mid \psi(\vec{\theta})\right\rangle\right|^{2}+\epsilon\left|\left\langle\psi_{1} \mid \psi(\vec{\theta})\right\rangle\right|^{2}$ 


\section{Optimization algorithm}

- Expensive to evaluate gradient of cost function

- Numerical differentiation, cost $\sim 0(n)$, where $n=$ number of parameters

- In contrast, for neural network, $\operatorname{cost~} \sim \mathrm{O}(\log n)$ by back propagation

- Preferable: gradient-free optimizer

- Noisy cost function

- Hardware fidelities, sampling error, ...

- Preferable: noise insensitive

- Local minimums

- Low energy but physically very different from the ground state (or targeted state)

- Preferable: global optimizer / knowledge to make reasonably good initial guess 


\section{Proof-of-principle expt. - Rabi model using Rigetti's device}

TLS

$\Omega \quad$ Rabi Hamiltonian: two-level system (TLS) coupled to a photon mode

$$
H=\omega a^{\dagger} a+\frac{\Omega}{2} \sigma_{z}+g\left(a^{\dagger}+a\right) \sigma_{x}
$$

Number-basis binary encoding:

Photon photon mode truncated to up to 3 photons

$$
\begin{aligned}
& |n=0\rangle=|00\rangle_{q}|n=1\rangle=|01\rangle_{q} \\
& |n=2\rangle=|10\rangle_{q}|n=3\rangle=|11\rangle_{q}
\end{aligned}
$$

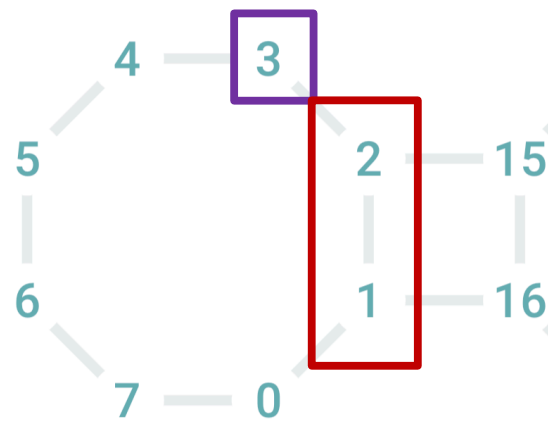

$14-13$

12

$$
\begin{aligned}
\mathrm{H}= & \omega \sigma_{0}^{z}+\frac{\omega}{2} \sigma_{1}^{z}+\frac{\Omega}{2} \sigma_{2}^{z}+g \sqrt{\sigma_{0}^{z}+2} \sigma_{1}^{x} \sigma_{2}^{x} \\
& +\frac{g}{\sqrt{2}} \sigma_{0}^{x} \sigma_{1}^{x} \sigma_{2}^{x}+\sigma_{0}^{y} \sigma_{1}^{y} \sigma_{2}^{x}+\frac{3 \omega}{2},
\end{aligned}
$$

$17-10$

11 


\section{Hardware efficient ansatz}

1Q-gate layer Entanglement-gate layers

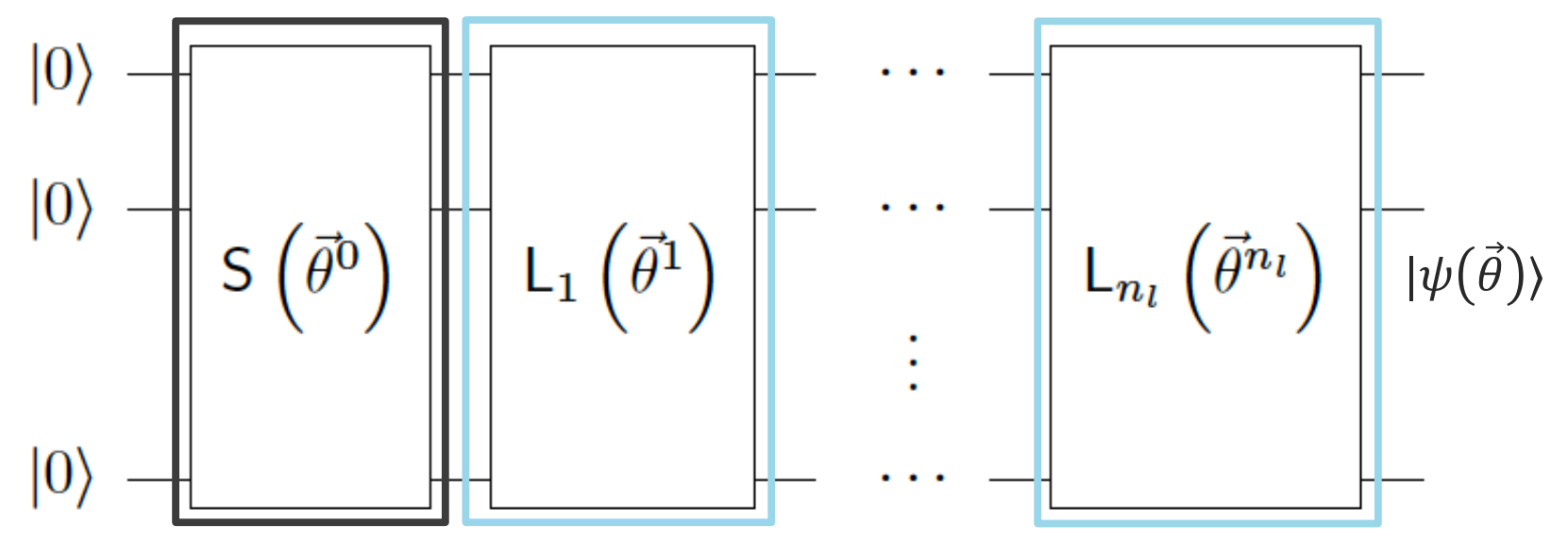

Ansatz consists only of native gates supported by the hardware

e.g. $\mathrm{R}_{\mathrm{Y}}(\theta), \mathrm{R}_{\mathrm{Z}}(\theta)$ and $\mathrm{CZ}$

3 qubits with 1 entanglement

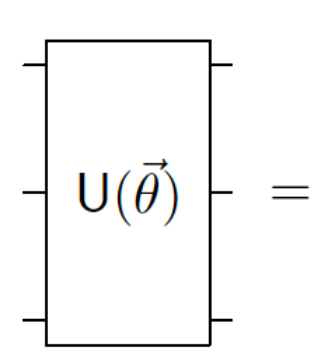

\begin{tabular}{|l|l|l|l|}
\hline $\mathrm{R}_{\mathrm{Y}}\left(\theta_{0}\right)$ & $\mathrm{R}_{\mathrm{Z}}\left(\theta_{1}\right)$ \\
$\mathrm{R}_{\mathrm{Y}}\left(\theta_{2}\right)$ & $\mathrm{R}_{\mathrm{Z}}\left(\theta_{3}\right)$ \\
$\mathrm{R}_{\mathrm{Y}}\left(\theta_{4}\right)$ & $\mathrm{R}_{\mathrm{Z}}\left(\theta_{5}\right)$ \\
\hline & $\mathrm{R}_{\mathrm{Y}}\left(\theta_{6}\right)$ & $\mathrm{R}_{\mathrm{Y}}\left(\theta_{8}\right)$ & $\mathrm{R}_{\mathrm{Z}}\left(\theta_{9}\right)$ \\
& & $\cdot \mathrm{R}_{\mathrm{Y}}\left(\theta_{10}\right)$ & $\mathrm{R}_{\mathrm{Z}}\left(\theta_{11}\right)$ \\
\hline & $\mathrm{R}_{\mathrm{Y}}\left(\theta_{12}\right)$ & $\mathrm{R}_{\mathrm{Z}}\left(\theta_{13}\right)$ \\
\hline
\end{tabular}




\section{Optimizers}

\begin{tabular}{|c|c|}
\hline Optimization algorithm & \\
\hline $\begin{array}{c}\text { Simultaneous Perturbation } \\
\text { Stochastic Approximation (SPSA) }\end{array}$ & Stochastic \\
\hline Nelder-Mead & Gradient-free \\
\hline $\begin{array}{c}\text { Constrained Optimization BY Linear } \\
\text { Approximations (COBYLA) }\end{array}$ & Gradient-free \\
\hline $\begin{array}{c}\text { Bound Optimization BY Quadratic } \\
\text { Approximation (BOBYQA) }\end{array}$ & Gradient-free \\
\hline $\begin{array}{c}\text { Covariance Matrix Adaptation } \\
\text { Evolution Strategy (CMA-ES) }\end{array}$ & Evolutionary algorithm: \\
\hline
\end{tabular}




\section{Optimizers with noisy device}

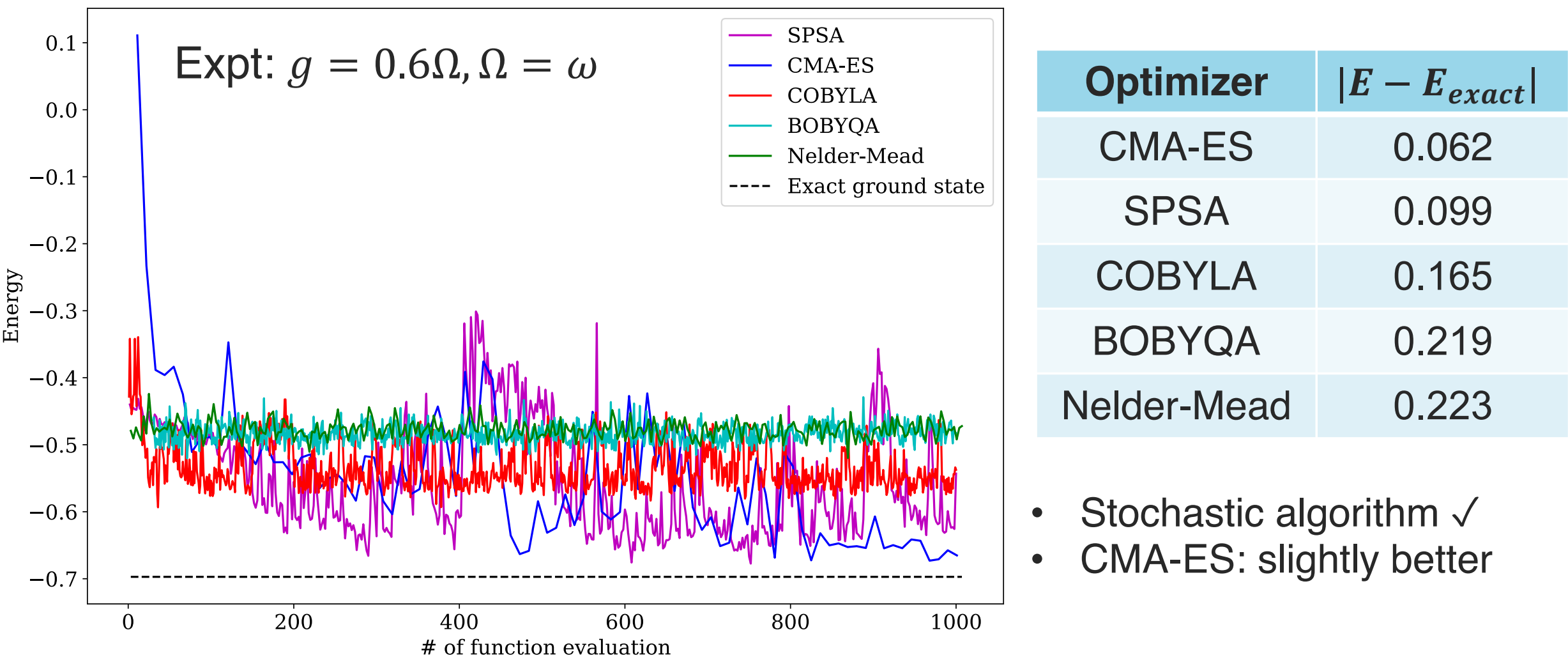




\section{Experimental result}

Error bars: sampling error of 200000 shots

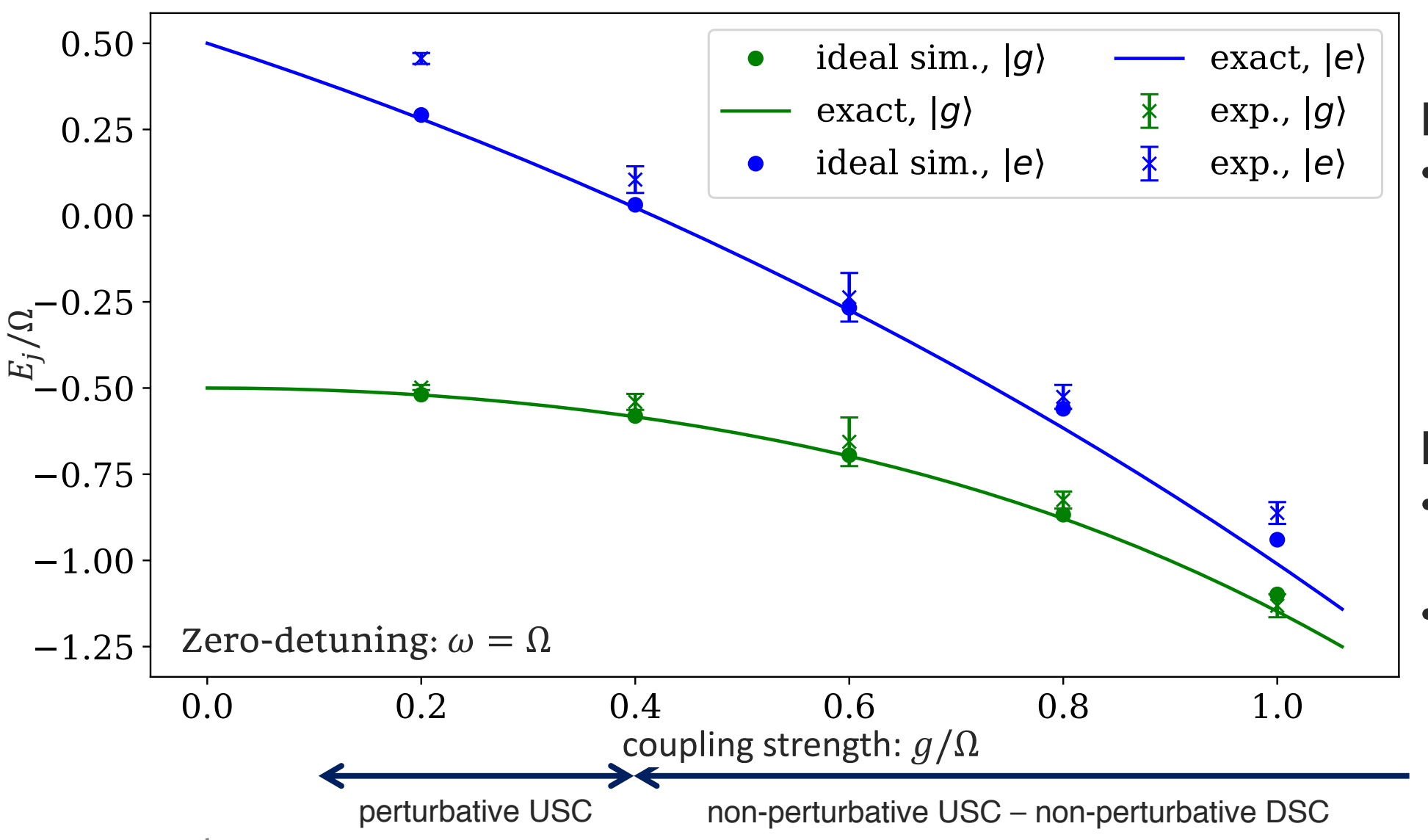

Energy gap

- Consistent trend across multiple parameter regimes

Deviation

- Hardware fidelities

- Photon cutoff for $g \geq 0.8 \Omega$ 


\section{Generalizing to bigger systems?}

- Proof-of-principle experiment of Rabi model

- 3-qubit implementation on Rigetti's device

- Error mitigation techniques

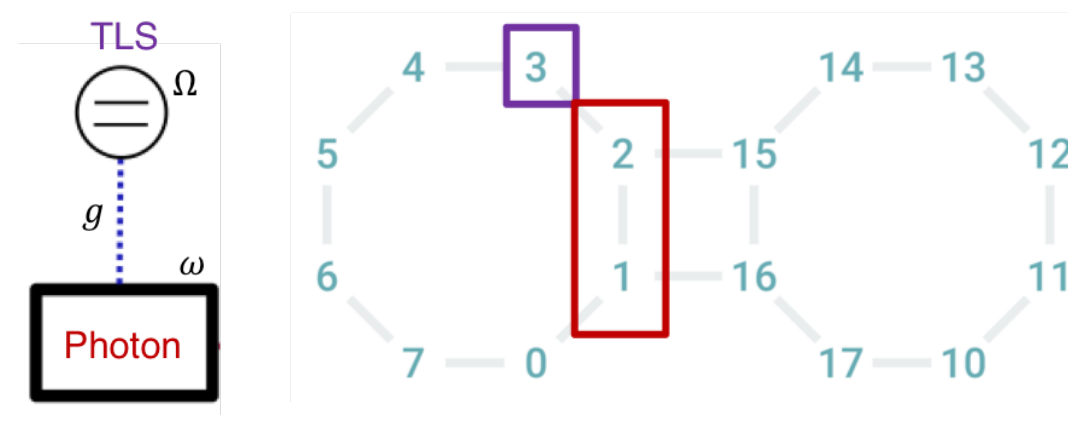

- Generalize to bigger system?
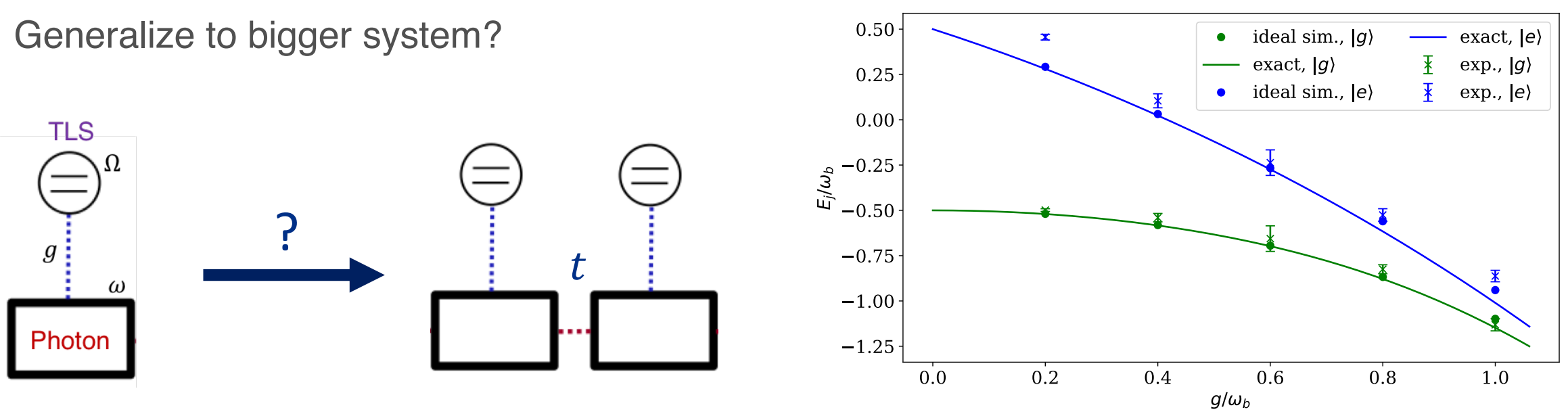


\section{Rabi dimer: hardware efficient ansatz}

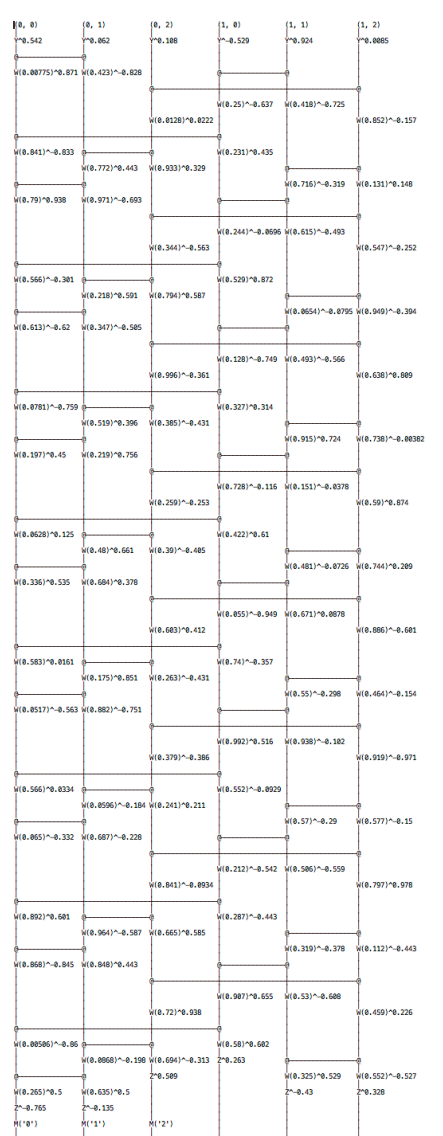

$$
\begin{aligned}
\mathrm{H}= & \frac{\omega}{2}\left(\mathrm{p}_{0}^{2}+\mathrm{x}_{0}^{2}+\mathrm{p}_{1}^{2}+\mathrm{x}_{1}^{2}\right)+t \mathrm{x}_{0} \mathrm{x}_{1} \\
& +g\left(\mathrm{x}_{0} \sigma_{0}^{x}+\mathrm{x}_{1} \sigma_{1}^{x}\right)+\frac{\Omega}{2}\left(\sigma_{0}^{z}+\sigma_{1}^{z}\right)
\end{aligned}
$$

\begin{tabular}{|c|c|}
\hline \# of parameters & 204 \\
\hline \# of 1Q gates & 110 \\
\hline \# of 2 Q gates & 49 \\
\hline Circuit depth & 51 \\
\hline
\end{tabular}

- Too many \# of optimization steps

- Large \# of local minima

Dimer: crossover between hopping and blockade of bosons
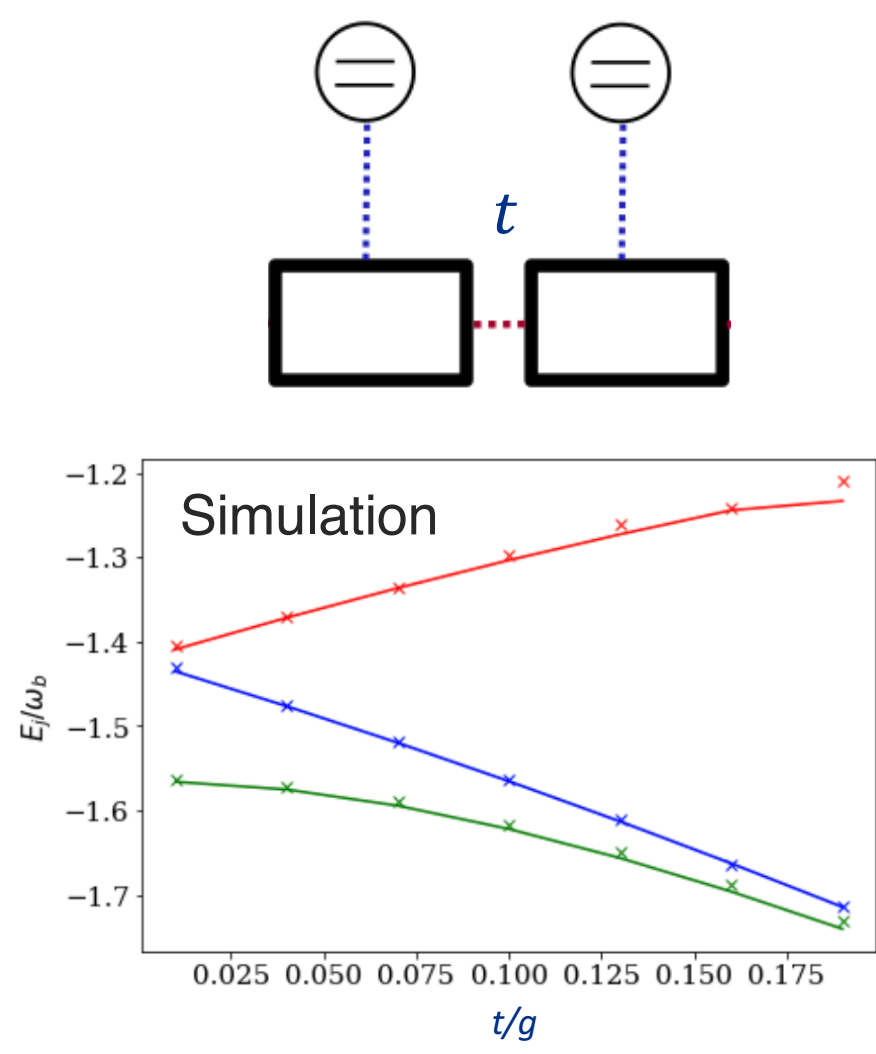


\section{Circuit depth of model-motivated ansatz}

\begin{tabular}{|c|c|}
\hline \multicolumn{2}{|c|}{ Per one 'Trotter step' } \\
\hline \# of parameters & 9 \\
\hline \# of 1Q gates & 24 \\
\hline \# of 2Q gates & 39 \\
\hline
\end{tabular}

Fidelity for 5 steps $0.999^{* *} 120 * 0.995^{\star *} 195$ $=0.33$
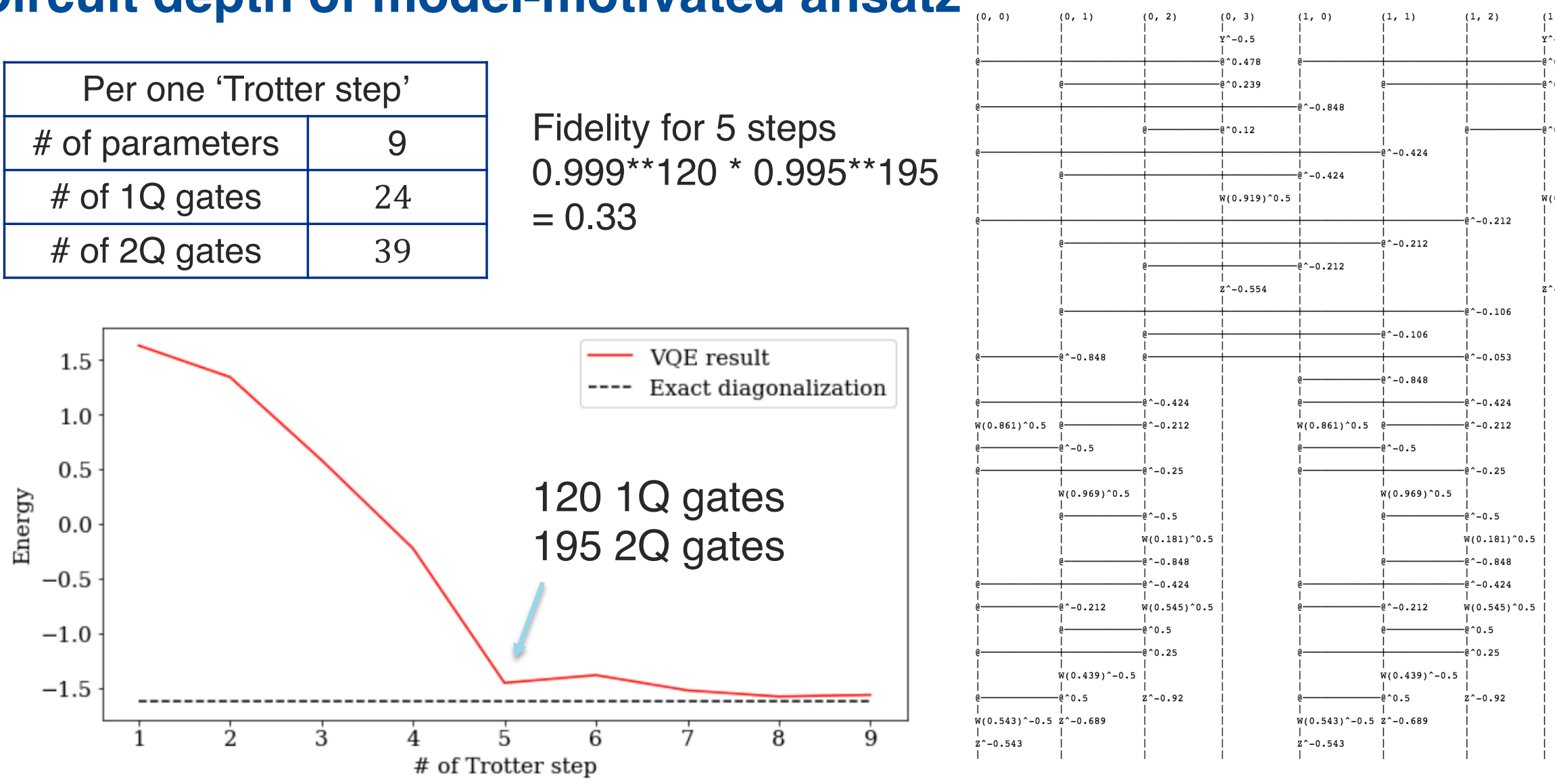


\section{Overview}

- VQE algorithms for interacting bosons

- Demonstrate with a 3-qubit implementation
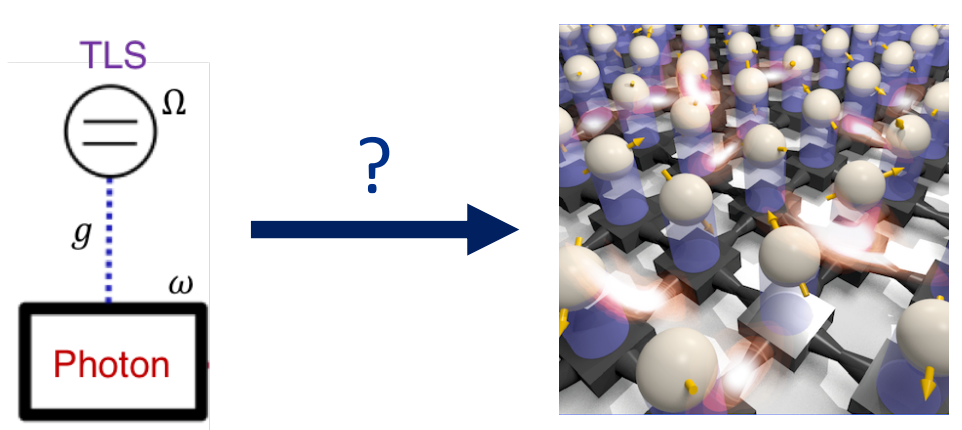

- Scalability:

- Hardware-efficient ansatz: large \# of parameters

- Explore model-motivated ansatz with less demanding circuit depth

- Optimizing a high-dimension cost function

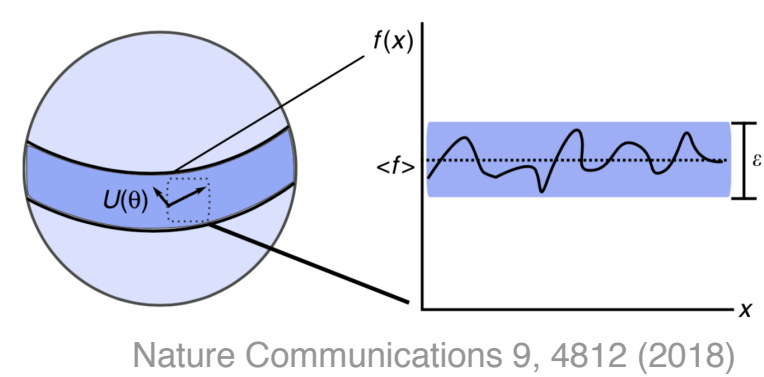

Hardware efficient

Model motivated

Increasing circuit depth

Increasing number of

optimization parameters 